\title{
Bidirectional Learning of Handwriting Skill in Human-Robot Interaction
}

\author{
Hang Yin \\ LASA, École Polytechnique \\ Fédérale de Lausanne \\ INESC-ID and Instituto Super \\ ior Técnico, Universidade de \\ Lisboa \\ hang.yin@epfl.ch
}

\author{
Aude Billard \\ LASA, École Polytechnique \\ Fédérale de Lausanne \\ aude.billard@epfl.ch
}

\author{
Ana Paiva \\ INESC-ID and Instituto Super \\ ior Técnico, Universidade de \\ Lisboa \\ ana.paiva@inesc-id.pt
}

\begin{abstract}
This paper describes the design of a robot agent and associated learning algorithms to help children in handwriting acquisition. The main issue lies in how to program a robot to obtain human-like handwriting and then exploit it to teach children. We propose to address this by integrating learning from demonstrations paradigm, which allows the robot to extract a task index from intuitive expert (e.g., adults) demonstrations. We present our work on the development of an algorithm, as well as its validation by learning compliant robotic writing motion from the extracted index. Also discussed is the synthesis of the learned task in the prospective work of transferring the task skill to users, especially in terms of learning by teaching. The undergoing work about the design of a sensor-embedded pen is introduced. This will be used as an intuitive interface for recording various handwriting related information in the interaction.
\end{abstract}

\section{Keywords}

Learning from demonstrations, human robot interaction, learning by teaching

\section{INTRODUCTION}

The development of children handwriting is extensively studied in the fields of education and occupational therapy [3], focusing on the evolution of handwriting characteristics (e.g., legibility, speed and tip force), as well as the effect of human intervention. However, substantial challenges exist for robots to efficiently acquire the knowledge for human-like handwriting, including what is the human-likeness (e.g., features of a legible letter) and how to control robotic writing (e.g., regulating contact force/disturbance). The knowledge is also fundamental to evaluate and instruct children in autonomous interaction. This motivates a bidirectional learning task between humans and robots (Figure 1). Learning

Permission to make digital or hard copies of part or all of this work for personal or classroom use is granted without fee provided that copies are not made or distributed for profit or commercial advantage, and that copies bear this notice and the full citation on the first page. Copyrights for third-party components of this work must be honored. For all other uses, contact the owner/author(s). Copyright is held by the author/owner(s).

HRI'15 Extended Abstracts, March 2-5, 2015, Portland, OR, USA.

ACM 978-1-4503-3318-4/15/03.

http://dx.doi.org/10.1145/2701973.2702718.

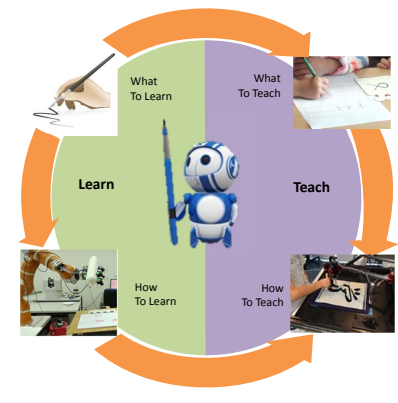

Figure 1: Bidirectional learning of handwriting: what/how to learn/teach

from demonstrations (a.k.a, imitation learning) is a fundamental framework for robots to learn through intuitive human interaction [1]. Our work is based on the implicit imitation learning, where a cost function that scores state and action is learned. This index accounts for an invariant task specification for both humans and robots, with respect to which, robots can evaluate users' handwriting, and generate feedback behavior accordingly. The interaction is supported by a pen-based interface, which collects multi-modal sensory information and intuitive user input.

\section{APPROACHES}

\subsection{Imitation learning: Energy-based Model}

The idea of implicit imitation learning is to extract a cost function $C(\boldsymbol{x}, \boldsymbol{\theta})$ from observed trajectory $\tau^{*}=\left\{\boldsymbol{x}_{t}^{*}\right\}$, where $\boldsymbol{\theta}$ is the parameter to infer and $\boldsymbol{x}$ denotes the state features (e.g., position of pen-tip in handwriting) which might subject to the constraint of dynamics and be indexed by time $t$. The hypothesis is that observed $\tau^{*}$ is optimal with respect to the unknown $C(\boldsymbol{x}, \boldsymbol{\theta})$, implies to find $\boldsymbol{\theta}^{*}$ such that

$$
\tau^{*}=\underset{\tau}{\operatorname{argmin}} J\left(\tau, \boldsymbol{\theta}^{*}\right)=\underset{\left\{\boldsymbol{x}_{t}\right\}}{\operatorname{argmin}} \sum_{t=1}^{T} C\left(\boldsymbol{x}_{t}, \boldsymbol{\theta}^{*}\right)
$$

The resulting problem is an ill-posed inverse optimal control problem. One elegant way to address it is to interpret $J(\tau, \boldsymbol{\theta})$ as the energy of trajectories and the ones with low energy are more likely to be observed. This accumulated 


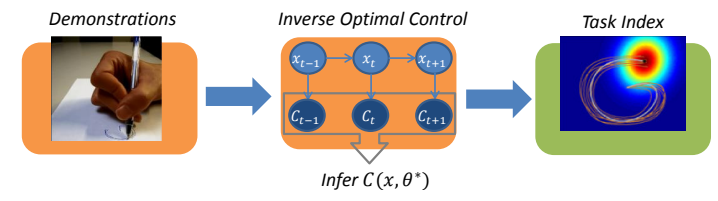

Figure 2: Learning task index from demonstrations

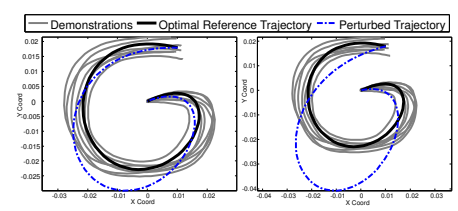

Figure 3: Left - observed human writing (blue dash); right - aggravation from perturbed model

cost parameterizes a Boltzmann distribution over the trajectories

$$
P(\tau \mid \boldsymbol{\theta})=\frac{\exp (-J(\tau, \boldsymbol{\theta}))}{\int_{\tau^{\prime}} \exp \left(-J\left(\tau^{\prime}, \boldsymbol{\theta}\right)\right)}
$$

Thus a regularized (1) can be revealed and the resulting model resolves ambiguity and is robust to noisy/suboptimal demonstrations. Also, $J(\tau, \boldsymbol{\theta})$ is of a general form so various feature or structure can be designed to synthesize the task; and generate behavior according to the original/variant goal;

\subsection{Task Synthesis - Learning Handwriting from Humans}

The robot learns handwriting by estimating the distribution parameter $\boldsymbol{\theta}$ from human demonstrations $\tau^{*}$. We propose to exploit an importance-sampling-like method [4], which offers flexibility of learning with nonlinear features and generating robot motion as a model-free method. The potential informative features include curvature-based features and their dependency across trajectories. These are desired to address the challenge of encoding multi-stroke letters in [2]. Figure 2 shows the general flow of learning the cost function for handwriting.

\subsection{Trajectory Generation - Teaching Hand- writing to Humans}

With the learned cost function, the robot can synthesize autonomous motion by incorporating learner's writing feedback. This can be achieved by perturbing the model to generate motion that exaggerates poorly written features. As is shown in Figure 3, the learner's writing is evaluated by extracting parameters' perturbation $\delta \boldsymbol{\theta}$. By amplifying this discrepancy, e.g., applying 2.5 times $\delta \boldsymbol{\theta}$, the robot can write a letter that aggravates the error for the learner to correct. Such approach exploits the learning by teaching paradigm to engage the learners and improve the learning gains. It is also similar to the learning from failures while the failed demonstrations are used in a different way.

\section{RESULTS AND PROPOSED WORK}

\subsection{Learning Compliant Handwriting Motion}

The task index encodes letter trajectories, and can also be used to develop motion compliance for safe and robust
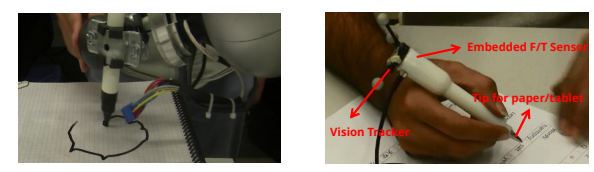

Figure 4: Robotic writing Figure 5: Force/torque motion and compliance sensor embedded pen
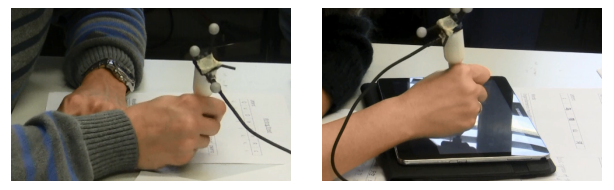

Figure 6: Writing with sensor-embedded pen - ball tip on paper and touch tip on a tablet

interaction. Concretely, a quadratic form of nonlinearly combined features $\left(C(\boldsymbol{x}, \boldsymbol{\theta})=\left(\boldsymbol{x}-\boldsymbol{x}_{t}^{\text {ref }}\right)^{T} \boldsymbol{Q}_{t}\left(\boldsymbol{x}-\boldsymbol{x}_{t}^{r e f}\right)\right.$, $\left.\boldsymbol{\theta}=\left\{\boldsymbol{x}_{t}^{r e f}, \boldsymbol{Q}_{t}\right\}\right)$ can be defined and learned. Deriving motion by optimizing this cost will lead to a varying gain linear feedback controller. The gain is associated with $\boldsymbol{Q}_{t}$ which encodes the desirability to track the target trajectory. Figure 4 shows the compliant behavior derived from the learned index and its consistency with the demonstration variability.

\subsection{Interface Development}

A sensor-embedded (e.g., force sensor) pen is under development. This will serve as an interface for collecting multimodal sensor input in writing interaction (Figure 5). The prototype is under evaluation in an adult handwriting experiment which includes writing on regular paper and tablet (Figure 6), where the tip force and motion features (e.g., variability) in different environments are investigated. It is possible to incorporate modules for other sensor readings, such as an inertial unit for measuring the pose of pen barrel.

\section{ACKNOWLEDGMENTS}

This work is partially funded by Swiss National Center of Robotics Research and national funds through Fundação para a Ciência e a Tecnologia (FCT) with reference UID/ CEC/50021/2013 and doctoral grant (SFRH/BD/51933/2012) under IST-EPFL Joint Doctoral Initiative.

\section{REFERENCES}

[1] S. Calinon, Z. Li, T. Alizadeh, N. G. Tsagarakis, and D. G. Caldwell. Statistical dynamical systems for skills acquisition in humanoids. In Proc. IEEE Intl Conf. on Humanoid Robots, pages 323-329, Osaka, Japan, 2012.

[2] D. Hood, S. Lemaignan, and P. Dillenbourg. When children teach a robot to write: An autonomous teachable humanoid which uses simulated handwriting. In Proc. of the ACM/IEEE Intl Conf. on Human-Robot Interaction, Portland, USA, 2015 (In press).

[3] Z. Jenny and W. Margaret. The development of graphomotor skills. In A. Henderson and C. Pehoski, editors, Hand function in the child: Foundations for remediation, 2nd Edition. Mosby, Inc, 2006.

[4] H. Yin, A. Paiva, and A. Billard. Learning cost function and trajectory for robotic writing motion. In Proc. IEEE Intl Conf. on Humanoid Robots, Madrid, Spain, 2014. 